\title{
Auditing face mask practice among the general public
}

\author{
Abeywardena HMW, Ekanayaka P, Kodithuwakku R
}

\section{Introduction}

COVID-19 is an infectious disease caused by a newly discovered coronavirus which spreads primarily through droplets. Airborne transmission of the virus can occur in health care settings through specific medical procedures, called aerosol generating procedures. Some outbreak reports related to indoor crowded spaces have suggested the possibility of aerosol transmission, combined with droplet transmission. Since there is no vaccination or specific treatment, prevention is the only way to interrupt the spread of infection by hand hygiene, not touching the face and with social distancing.

Since there is a possibility of transmission from pre- symptomatic patients, it is advised to use fabric masks in public places where community transmission may occur. However, knowledge on the correct way of wearing masks is limited. This audit was done to evaluate the knowledge of the general public on wearing fabric masks.

\section{Methods}

A cross-sectional study was done based on 5 minute interview based information collected from patients visiting outpatient clinics at District General Hospital, Matale, after obtaining verbal consent. The indication, type and way of wearing fabric masks were obtained from the study sample. Re-audit was done on the same day after educating patients on the correct way of wearing masks.

\section{Results}

Of 83 patients, 76 were wearing fabric masks and 7 wore medical masks at the time of the audit. Of the 83, 80 thought the masks were for their safety, and 14 wore the masks for their own safety and for the safety of others. Two wore the masks in obedience to the regulations of the country. Of the 83, 46 were wearing the masks incorrectly, either leaving nose or nose and mouth exposed. Thirty four patients touched the front of their masks at least once, during the interview.

Following demonstration and education on correct wearing of the mask, of the 83,53 wore the masks correctly and touching of the masks was reduced to 11 persons during the interview. However, 78 continued to believe that wearing a mask was for their own protection.

\section{Conclusions}

From this small sample, it appears that the general public has a very limited knowledge on the use of face masks, including the indication and correct wear. This needs to be improved by frequent awareness programmes as it has a vital importance in protecting against community spread of Covid-19.,

Keywords: COVID-19, face masks, audit 